\title{
Prevalence of pathological myopia among patients in a large tertiary care center in Egypt
}

\author{
Ayman Gehad Elnahry*, Mohamed Mahmoud Khafagy, Soheir Mohamed Esmat and Hassan Aly Mortada \\ Department of Ophthalmology, Faculty of Medicine, Cairo University, Cairo, Egypt
}

\begin{abstract}
Purpose: To determine the prevalence of Pathological Myopia at the general ophthalmology clinic of our University Hospital over a period of 3 months.

Methods: This is a cross sectional study to determine the prevalence of Pathological Myopia at the ophthalmology clinic of our University Hospital. Patients had full ophthalmological examination. Eyes were subjected to non-cycloplegic refraction using an autorefractometer. Eyes not liable for refracton (e.g. vitreous hemorrhage) underwent either A scan or B scan ultrasonography to determine their axial length. The prevalence of Pathological Myopia in this sample of eyes was then determined, where Pathological-Myopia was defined as refraction more than or equal to $-6 \mathrm{D}$ spherical equivalent (SphEq) or an axial length more than or equal to $25.5 \mathrm{~mm}$.

Results: A total of 1289 eyes of 668 consecutive patients were examined. One hundred and forty eyes (10.9\%) were found to have Pathological-Myopia. The number of patients with at least one pathologically myopic eye was 84 patients (12.6\% of examined patients). Of these patients, 19 (22.6\%) had unilateral PathologicalMyopia, while $65(77.4 \%)$ had bilateral Pathological-Myopia. The refractive error of pathologically myopic eyes ranged from $-3.00 \mathrm{D}$ to $-26.00 \mathrm{D}$ (mean $-12.11 \pm$ 5.05D) calculated as the SphEq of their refraction. Their axial lengths ranged from $25.50 \mathrm{~mm}$ to $36.00 \mathrm{~mm}$ (mean $28.65 \pm 2.34 \mathrm{~mm}$ ).
\end{abstract}

Conclusion: This study suggests a high prevalence of Pathological Myopia in Egypt compared to studies from other countries. A large community-based study in Egypt is required to determine the prevalence in the general population.

Keywords: Pathological Myopia, axial length, prevalence, refractive error, Egypt

\section{Introduction}

Pathological myopia is a major growing health problem worldwide [1] that is particularly prevalent in Asian and Middle Eastern countries [2]. Pathological myopia, high myopia and degenerative myopia are various terms used to describe this condition. In 1970, Duke Elder defined pathological myopia as myopia associated with degeneration of the sclera, choroid, and retinal pigment epithelium, with visual function compromise, [3] while in 1988 Tokoro defined it by excessive and progressive elongation of the axial length [4]. Other studies have defined pathological myopia as myopia more than or equal to $-6 \mathrm{D}$ or an axial length of more than $25.5 \mathrm{~mm}$ [5]. Yet, there has not been a standard definition for pathological myopia to date [6].

It has emerged as a major health concern for several reasons. First, in developed countries in East and Southeast Asia, as Singapore, China and Taiwan, the prevalence of myopia has rapidly increased in the last 50-60 years [7]. In these countries, $80-90 \%$ of children finishing high school are now myopic, with $10-20 \%$ being highly myopic [8]. In a study of 5060 students in a Chinese university, $95.5 \%$ were found to be myopic (Spherical equivalent $(\mathrm{SphEq})<-0.50 \mathrm{D})$, while $19.5 \%$ were highly myopic $(\mathrm{SphEq}<-6.00 \mathrm{D})$. Only $3.3 \%$ were emmetropic [9]. Meanwhile, a Taiwanese eye study showed an increase in prevalence of high myopia among first year university students from $23.5 \%$ in 1988 , to $38.4 \%$ in 2005 [10]. The prevalence is also increasing in the United States [11]. In the USA, burden of disease studies has been carried out on myopia. It has been estimated that 2 billion US dollars per year in 1983 and 4.6 billion US dollars per year in 1994 were spent in the USA on correcting myopic refractive errors [12]. A study of 12,010 participants in the US showed that the prevalence of myopia (SphEq
$<-0.50 \mathrm{D})$ for ages $20-39,40-59$ and $>60$ years was $50.2 \%, 50.1 \%$ and $26.5 \%$ respectively, while the prevalence for severe myopia $(\mathrm{SphEq}<$ $-5.00 \mathrm{D}$ ) for ages $20-39,40-59$ and $>60$ years was $7.4 \%, 7.8 \%$ and $3.1 \%$ respectively [13]. It is estimated that 153 million people over 5 years of age globally have visual impairment due to uncorrected myopia and other refractive errors [14].

Second, people with high myopia are at greater risk of potentially blinding myopic complications. In fact, pathological myopia is now one of the leading causes of legal blindness in many developed countries [15]. In the USA, it is the seventh leading cause of blindness, [16] while according to a recent study from Japan, pathological myopia was the third leading cause of bilateral vision loss and the leading cause of monocular blindness in that population [17] In Canada it is estimated that $9 \%$ of blindness was due to pathological myopia, [18] while in the Beijing Eye Study, it was the most frequent cause of low vision and blindness in subjects 40 to 49 years old [19].

In Egypt, many patients are found to have high myopia. In 1960, Fuchs mentioned that the prevalence of pathological myopia in Egypt was $0.2 \%$, [20] while Gawdat, in 1976, showed that it is $7.4 \%$ [21]. This

${ }^{\star}$ Correspondence to: Ayman Elnahry, Department of Ophthalmology, Faculty of Medicine, Cairo University, Cairo, Egypt 11956, Tel: +20-1224927604; E-mail ayman_elnahri@hotmail.com

Key words: pathological myopia, axial length, prevalence, refractive error, Egypt

Received: October 05, 2018; Accepted: October 22, 2018; Published: October 26,2018 
indicates an increasingly higher prevalence of pathological myopia among Egyptians over time. In 2015, Massoud and Nassr showed it to be present in $10.8 \%$ of university students [22]. In this study, we sought to determine the prevalence of Pathological Myopia in patients attending our University Hospital Ophthalmology clinic.

\section{Materials and methods}

This study is a cross-sectional study of a clinical population attending the Cairo University Hospital general ophthalmology clinic of our department in the period from September 2015 to November 2015. Ethical approval was obtained from the Institutional Review Board and written informed consent was obtained from all participants. The study adhered to the guidelines of the Declaration of Helsinki. Eyes which have undergone operations for posterior segment diseases, such as conventional RD surgery, vitrectomy and intravitreal injections were excluded. The study included 1289 eyes of 668 patients. There were 418 females and 250 males. Each eye had full ophthalmological examination including best corrected visual acuity (BCVA), intraocular pressure (IOP) measurement, anterior and posterior segment slit lamp biomicroscopy and fundus examination with indirect ophthalmoscopy. Eyes were subjected to non-cycloplegic refraction using an autorefractometer (Topcon Inc, Tokyo, Japan). Eyes not liable for refracton (e.g. dense cataracts or vitreous hemorrhage) underwent either A scan or B scan ultrasonography to determine their axial length. Those eyes found to be myopic with refraction more than or equal to $-6 \mathrm{D}$ spherical equivalent (SphEq) or have an axial length more than or equal to $25.5 \mathrm{~mm}$ were diagnosed as pathologically myopic eyes. Spherical equivalent was calculated as the spherical value of the refractive error plus half of the cylindrical value.

Regarding B scan ultrasonography, the Sonomed B 3000 (Escalon, Ardmore, PA, USA) model was used. We used the $10 \mathrm{MHz}$ probe and recorded the axial length, the presence of staphylomata, vitreal opacities, posterior vitreous detachment and retinal detachment. The Sonomed A scan ultrasonography PacScan 300A (Escalon, Ardmore, PA, USA) was used to measure the axial length of included eyes. Five scans were done for each eye and the average value was documented.

\section{Statistical analysis}

All analyses were done using IBM SPSS v20.0 statistical software (IBM Corporation, NY, USA). Descriptive statistics was calculated, and the data was summarized as mean \pm SD for numerical data, and as frequencies and percentages for categorical data.

\section{Results}

We examined 1289 eyes of 668 consecutive patients who met the inclusion criteria. The number of female patients were 418 (62.6\%), while 250 patients (37.4\%) were males. The age range was $14-84$ years (mean $41 \pm 15$ years). Of the 1289 eyes examined, 140 were found to be pathologically myopic, which represents $10.9 \%$ of examined eyes (Figure 1). The number of patients with at least one pathologically myopic eye was 84 patients (12.6\%) out of the 668 examined patients. Forty-five (53.6\%) were females and 39 (46.4\%) were males. Of these patients with pathologically myopic eyes, 19 had unilateral pathologic myopia (22.6\%) while 65 had bilateral pathologic myopia (77.4\%).

The age of pathologically myopic patients ranged from 15 to 75 years (mean $42 \pm 15$ years). Their refractive error ranged from $-3.00 \mathrm{D}$ to $-26.00 \mathrm{D}$ (mean $-12.11 \pm 5.05 \mathrm{D}$ ) calculated as the spherical equivalent of their refraction. Their axial length ranged from $25.50 \mathrm{~mm}$ to $36.00 \mathrm{~mm}$ (mean $28.65 \pm 2.34 \mathrm{~mm}$ ). One patient, with $-3.00 \mathrm{D}$ refractive error, was

\section{Eyes}

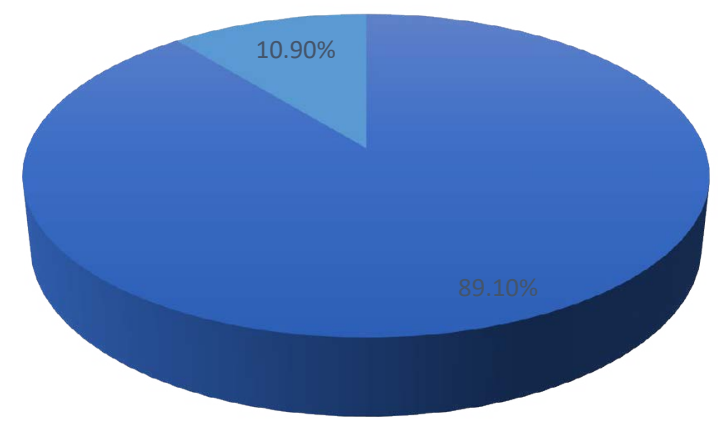

- Others Pathological Myopia

Figure 1. Prevalence of Pathological Myopia in eyes examined in our study

included in the study as he was clearly a pathologically myopic patient with axial length more than $27.00 \mathrm{~mm}$ and posterior staphyloma. Further examinations revealed relatively flat corneas in both eyes (Keratometric readings below 40D).

\section{Discussion}

Our study showed that the prevalence of Pathologically Myopic eyes was $10.9 \%$ in our University Hospital's general ophthalmology clinic. Regarding patients with pathologically myopic eyes, $22.6 \%$ had unilateral pathological myopia, while $77.4 \%$ had bilateral pathological myopia.

Several studies have reported the prevalence of Pathological Myopia (PM) in studied subjects. In a study in 2002 in Sydney, Australia, only $2.7 \%$ of patients were found to be highly myopic [23]. This study, however, was a population based survey in an urban population aged 49 years and older, and high myopia was defined as SphEq more than or equal to $-5.00 \mathrm{D}$ in at least one eye, in contrast to our study which was a study of a clinical population of patients, many of whom were from rural areas of Egypt with age range of 15-75 years and with high myopia defined as SphEq more than or equal to -6.00D. Our higher prevalence might be explained by the fact that we used a clinical population, where high myopia might be more common than in a general population due to the morbidity associated with high myopia, and the younger age of patients included in our study, with pathological myopia becoming more prevalent in the newer generations according to many studies, [10] however we used a higher cut-off value to define PM and many of our patients were from rural areas which is known to be less myopigenic than urban areas.

In a study by Katz et al. in 1997, refractive error was measured in a population-based sample of black and white adults aged 40 or older residing in east Baltimore, USA. The prevalence of high myopia in black adults was $0.8 \%$ while it was $1.8 \%$ in white adults [24]. This study, however, excluded aphakic eyes. It is important to note that this study was performed between 1985 and 1988, when intraocular lens implantation following cataract surgery was just starting to be routinely performed. The majority of patients who have undergone cataract surgery by then were thus probably aphakic and excluding them might result in underestimation of the prevalence of high myopia as high 
myopes are more liable to undergo cataract surgery due to refractive reasons, as well as increased risk of cataract formation. Pseudophakic and aphakic patients however were included in our study through the measurement of their axial length, which we thought could result in a more accurate estimation of the prevalence of high myopia. Of the 127 highly myopic eyes examined in our study, $12.6 \%$ were either pseudophakic or aphakic and $10.2 \%$ could not be refracted using the autorefractometer used in our study.

In that study by Katz et al in 1997, people of different ethnicities were found to have different prevalence of high myopia despite living in a similar area and having a similar level of education most probably due to genetic and behavioral differences between people of different ethnicities [24]. This might suggest that our much higher prevalence might also be due to these differences.

In the Beijing eye study, a population-based study in Greater Beijing, China, the prevalence of high myopia in examined eyes was found to be $2.4 \%$, however, all pseudophakic and aphakic eyes were excluded as well as patients who had undergone refractive surgery, [25] which might have underestimated the prevalence of high myopia. This study was divided into a rural part and an urban part with $46.1 \%$ of patients from rural areas and $53.9 \%$ from urban areas. High myopia was more prevalent in urban areas. In the Hisayama study in Japan, $12.1 \%$ of eyes had an axial length more than or equal to $25.00 \mathrm{~mm}$ and $5.5 \%$ had an axial length more than or equal to $26.00 \mathrm{~mm}$ [26]. In our study, pathological myopia was defined as an axial length more than or equal to $25.50 \mathrm{~mm}$. In this study, pseudophakics as well as patients without refraction data were not excluded.

In a study by Hsiang et al in 2008 in Tokyo, Japan, high myopia was defined as axial length more than or equal to $26.50 \mathrm{~mm}$, [27] while in a study by Grossniklaus and Green in 1992, high myopia was defined as axial length more than or equal to $25.50 \mathrm{~mm}$ [5]. This indicates that the definition of high myopia, with regards to axial length, differs between studies and that the definition may differ between people of different ethnicities.

In a study by Fuchs in 1960, the prevalence of pathological myopia in Egypt was mentioned to be $0.2 \%$, [20] while in a study by Gawdat in 1976, the prevalence of high myopia was 7.4\% [21]. This might indicate an increasing prevalence of pathological myopia in Egypt over time as that found in other developing countries due to urbanization and increasing level of education and near work. In a study by Massoud and Nassr in 2015 to determine the prevalence of refractive errors among students of the University of Assiut, Egypt, myopia more than or equal to $-6 \mathrm{D}$ spherical equivalent was found in $10.8 \%$ of students [22]. This was equal to the prevalence found in our study, however the mean age of study participants (18 \pm 1.56 years) was much lower than our study.

Regarding pathologically myopic patients in our study, we noticed that $22.6 \%$ of patients had unilateral pathological myopia, while $77.4 \%$ had bilateral pathological myopia. In a community-based study performed in Hong Kong in 2008, 16.3\% of patients had unilateral pathological myopia while $83.7 \%$ had bilateral pathological myopia [28]. More studies comparing demographics and characteristics of unilateral and bilateral pathological myopias are needed as this might provide insights in the development or progression of Pathological Myopia.

Strengths in our study include the use of axial length measurement in addition to refraction to diagnose Pathological Myopia allowing a limited exclusion criterion with inclusion of pseudophakic and aphakic patients as well as patients with media opacities through the measurement of their axial length. This might have helped determine the true prevalence.
Limitations of our study include the use of a clinical population, where pathological myopia might be more prevalent. Absence of a standardized definition for Pathological Myopia limited our ability to compare results with other studies. The use of non-cycloplegic refraction might have overestimated the prevalence of Pathological Myopia due to the effect of accommodation

This study confirmed our suspicion that the prevalence of Pathological Myopia in our University Hospital's General Ophthalmology clinic is high compared to the prevalence found in studies from other countries. With Pathological Myopia being a potentially blinding condition, this has serious implications. A population-based study with a larger sample size, to determine the prevalence of Pathological Myopia in the general population in Egypt is therefore needed to estimate the true burden of the disease in this country.

\section{References}

1. Tano Y (2002) Pathologic myopia: where are we now? Am J Ophthalmol 134: 645-660. [Crossref]

2. Curtin B (1985) Basic science and clinical management. In: Curtin B, editor. The myopias. Philadelphia: Harper \& Row 237-245.

3. Duke-Elder S (1970) Pathological Refractive Errors. Duke-Elder S, ed. St Louis: Mosby 297-373

4. Tokoro T (1988) On the definition of pathologic myopia in group studies. Acto Ophthalmol Suppl 185: 107-108. [Crossref]

5. Grossniklaus HE, Green WR (1992) Pathologic findings in pathologic myopia. Retina 12: 127-133. [Crossref]

6. Beuerman RW (2010) Saw SM, Tan DTH, Wong TY, eds. Myopia: Animal models to clinical trials. Singapore: World Scientific 3-21.

7. Morgan I, Rose K (2005) How genetic is school myopia? Prog Retin Eye Res 24: 1-38. [Crossref]

8. Lin LL, Shih YF, Hsiao CK, Chen CJ (2004) Prevalence of myopia in Taiwanese schoolchildren: 1983 to 2000. Ann Acad Med Singapore 33: 27-33. [Crossref]

9. Sun J, Zhou J, Zhao P, Lian J, Zhu H, et al. (2012) High prevalence of myopia and high myopia in 5060 Chinese university students in Shanghai. Invest Ophthalmol Vis Sci 53: 7504-7509. [Crossref]

10. Wang TJ, Chiang TH, Wang TH, Lin LL, Shih YF (2009) Changes of the ocular refraction among freshmen in National Taiwan University between 1988 and 2005. Eye (Lond) 23: 1168-1169. [Crossref]

11. Vitale S, Sperduto RD, Ferris FL 3rd (2009) Increased prevalence of myopia in the United States between 1971-1972 and 1999-2004. Arch Ophthalmol 127: 1632-1639. [Crossref]

12. Javitt JC, Chiang YP (1994) The socioeconomic aspects of laser refractive surgery. Arch Ophthalmol 112: 1526-1530. [Crossref]

13. Vitale S, Ellwein L, Cotch MF, Ferris FL 3rd, Sperduto R (2008) Prevalence of refractive error in the United States, 1999-2004. Arch Ophthalmol 126: 1111-1119. [Crossref]

14. Resnikoff S, Pascolini D, Mariotti SP, Pokharel GP (2008) Global magnitude of visual impairment caused by uncorrected refractive errors in 2004. Bull World Health Organ 86: 63-70. [Crossref]

15. Young TL (2009) Molecular genetics of human myopia: an update. Optom Vis Sci 86 E8-8E22. [Crossref]

16. Alexander LJ (1994) Primary care of the posterior segment. Appleton \& Lange, Connecticut.

17. Iwase A, Araie M, Tomidokoro A, Yamamoto T, Shimizu H, et al. (2006) Prevalence and cause of low vision and blindness in a Japanese adult population: The Tajimi Study. Ophthalmology 113: 1354-1362. [Crossref]

18. Blacharski PA (1988) Pathologic progressive myopia, in DA Newsome Ed, Retina dystrophies and degenerations. Raven Press, New York 257-269. 4.

19. Xu L, Wang Y, Li Y, Wang Y, Cui T, et al. (2006) Causes of blindness and visual impairment in urban and rural areas in Beijing: the Beijing Eye Study. Ophthalmology 113: 1134. [Crossref] 
20. FUCHS A (1960) Frequency of myopia gravis. Am J Ophthalmol 49: 1418-1419. [Crossref]

21. Gawdat I (1976) Studies on the incidence of refractive errors in Egypt. Bull Ophthalmol Soc Egypt 69: 513-520. [Crossref]

22. Massoud MS, Nassr MA (2015) Refractive errors among students enrolled in Assiut University, Egypt. J Egypt Ophthalmol Soc 108: 21-25.

23. Vongphanit J, Mitchell P, Wang JJ (2002) Prevalence and progression of myopic retinopathy in an older population. Ophthalmology 109: 704-711. [Crossref]

24. Katz J, Tielsch JM, Sommer A (1997) Prevalence and risk factors for refractive error in an adult inner city population. Invest Ophthalmol Vis Sci 38: 334-340. [Crossref]
25. Liu HH, Xu L, Wang YX, Wang S, You QS, et al. (2010) Prevalence and progression of myopic retinopathy in Chinese adults: the Beijing Eye Study. Ophthalmology 117 1763-1768. [Crossref]

26. Asakuma T, Yasuda M, Ninomiya T, Noda Y, Arakawa S, et al. (2012) Prevalence and risk factors for myopic retinopathy in a Japanese population: the Hisayama Study. Ophthalmology 119: 1760-1765. [Crossref]

27. Hsiang HW, Ohno-Matsui K, Shimada N, Hayashi K, Moriyama M, et al. (2008) Clinical characteristics of posterior staphyloma in eyes with pathologic myopia. $\mathrm{Am} \mathrm{J}$ Ophthalmol 146: 102-110. [Crossref]

28. Lai TY, Fan DS, Lai WW, Lam DS (2008) Peripheral and posterior pole retinal lesions in association with high myopia: a cross-sectional community-based study in Hong Kong. Eye (Lond) 22: 209-213. [Crossref]

Copyright: $@ 02018$ Elnahry AG. This is an open-access article distributed under the terms of the Creative Commons Attribution License, which permits unrestricted use, distribution, and reproduction in any medium, provided the original author and source are credited. 